\title{
Three-Dimensional Topography of Corticopontine Projections from Rat Barrel Cortex: Correlations with Corticostriatal Organization
}

\author{
Trygve B. Leergaard, ${ }^{1}$ Kevin D. Alloway, ${ }^{2}$ Joshua J. Mutic, ${ }^{2}$ and Jan G. Bjaalie ${ }^{1}$ \\ ${ }^{1}$ Department of Anatomy, Institute of Basic Medical Sciences, University of Oslo, N-0317 Oslo, Norway, and ${ }^{2}$ Pennsylvania \\ State University, Department of Neuroscience and Anatomy, Hershey, Pennsylvania 17033
}

\begin{abstract}
Subcortical re-entrant projection systems connecting cerebral cortical areas with the basal ganglia and cerebellum are topographically specific and therefore considered to be parallel circuits or "closed loops." The precision of projections within these circuits, however, has not been characterized sufficiently to indicate whether cortical signals are integrated within or among presumed compartments. To address this issue, we studied the first link of the rat cortico-ponto-cerebellar pathway with anterograde axonal tracing from physiologically defined, individual whisker "barrels" of the primary somatosensory cortex (SI). The labeled axons in the pontine nuclei formed several, sharply delineated clusters. Dual tracer injections into different SI whisker barrels gave rise to partly overlapping, paired clusters, indicating somatotopic specificity. Three-dimensional reconstructions revealed that the clusters were components of concentrically organized lamellar subspaces. Whisker barrels in the same row projected to different pontine lamellae (side by side), the soma-
\end{abstract}

totopic representation of which followed an inside-out sequence. By contrast, whisker barrels from separate rows projected to clusters located within the same lamellar subspace (end to end). In the neostriatum, this lamellar topography was the opposite, with barrels in the same row contacting different parts of the same lamellar subspace (end to end). The degree of overlap among pontine clusters varied as a function of the proximity of the cortical injections. Furthermore, corticopontine overlap was higher among projections from barrels in the same row than among projections from different whisker barrel rows. This anisotropy was the same in the corticostriatal projection. These findings have important implications for understanding convergence and local integration in somatosensory-related subcortical circuits.

Key words: 3-D reconstruction; basal ganglia; cerebellum; cerebrocerebellar; double anterograde tracing; parallel circuits; pontine nuclei; somatosensory maps; somatotopy
Neurons in the cerebral cortex project to a number of subcortical targets. Many of these neurons belong to two major corticosubcortical re-entrant circuits, one including the basal ganglia (for review, see Heimer et al., 1995; Parent and Hazrati, 1995) and another including the pontine nuclei and cerebellum (for review, see Brodal and Bjaalie, 1992, 1997; Schmahmann and Pandya, 1997). Structural and functional specification have been studied extensively in the first links of these circuits, i.e., in the projections from the cerebral cortex to the pontine nuclei (Brodal, 1968, 1978; Mihailoff et al., 1978, 1985; Wiesendanger and Wiesendanger, 1982; Bjaalie and Brodal, 1989; Leergaard et al., 2000) and the neostriatum (Webster, 1961; Malach and Graybiel, 1986; Gerfen, 1989; Alloway et al., 1999). During development, the global topography of corticopontine projections appears to be determined by simple temporal and spatial gradients operative within source (cerebral cortex) and target (pontine nuclei) region (Leergaard et al., 1995). In agreement with these topographic principles, we recently demonstrated that the adult pontine projections from primary somatosensory cortex (SI) are systematically organized in a clustered threedimensional (3-D) map (Leergaard et al., 2000). Thus, the terminal fields of axons originating in different SI body part representations (perioral region, trunk, extremities) are largely segregated and preserve the somatotopic order of the cortical map. One interpretation of this arrangement would be that there are separate channels through the pontine nuclei for different major body representations of SI. Such segregated projection systems, connecting

\footnotetext{
Received May 12, 2000; revised Aug. 17, 2000; accepted Aug. 29, 2000.

Financial support was provided by European Community Grant Bio4 CT98-0182 to J.G.B., The Research Council of Norway, The Norwegian Academy of Sciences, and National Institutes of Health Grant NS37532 to K.D.A. We thank Annabjørg Bore and Christian Pettersen for expert technical assistance, Petter Laake for helpful assistance with the statistical analysis, and James M. Bower and Per Brodal for valuable comments on this manuscript.

Correspondence should be addressed to Dr. Jan G. Bjaalie, Department of Anatomy, Institute of Basic Medical Sciences, University of Oslo, P.O. Box 1105 Blindern, N-0317 Oslo, Norway. E-mail: j.g.bjaalie@basalmed.uio.no.

Copyright (C) 2000 Society for Neuroscience $0270-6474 / 00 / 208474-11 \$ 15.00 / 0$
}

cerebral cortical areas with the cerebellum, have previously been reported in the monkey (Hoover and Strick, 1999) and have prompted hypotheses of parallel pathways ("closed loops") in the cerebrocerebello-thalamocortical re-entrant circuits [for review and comparison with basal ganglia circuitry, see Middleton and Strick (2000)]. The detailed organization of pontine projections from neighboring cortical sites within the same SI body part representation is not known. Projections at this finer level could either maintain topographic specificity and segregation or, alternatively, allow structural convergence and functional integration, comparable with the assumed integration in the neostriatum of functionally related signals originating from separate SI cortical sites [for general considerations on parallel basal ganglia circuitry, see Alexander et al. (1986, 1990), Alexander and Crutcher (1990), and Alloway et al. (1999)].

To test these hypotheses, we have analyzed the small-scale topography of corticopontine projections from the orderly representation of mystacial vibrissae in SI (Woolsey and Van der Loos, 1970; Welker, 1976). In the present study, we have mapped the 3-D pattern of corticopontine projections in rats that received injections of two anterograde tracers in different SI whisker "barrels." We report that corticopontine projections from individual barrels are topographically organized. Our findings also suggest that pontine neurons can integrate functionally related signals from neighboring cortical sites. Because most of these rats had also been used to characterize the organization of corticostriatal projections (Alloway et al., 1999), we compared the relative patterns of corticostriatal and corticopontine projections from the same cortical sites. This comparison revealed several important principles concerning the comparative organization of these major corticofugal pathways.

\section{MATERIALS AND METHODS}

Surgical procedures and electrophysiological mapping. Thirteen adult male Sprague Dawley rats were used (see Table 1). All animal procedures were approved by an institutional animal welfare committee and were in compliance with National Institutes of Health guidelines for the use and care of laboratory animals. 
Figure 1. A, Drawing of the right cerebral hemisphere of the rat with a cartoon representation of the SI somatotopic map and the SI barrel cortex (redrawn from Welker, 1971, with permission). B, Drawing of somatotopically organized SI barrels representing the mystacial vibrissae (arranged in rows $A-E$, corresponding to the arrangement of vibrissae on the contralateral mystacial pad), as they appear in CO-stained sections (redrawn from Fabri and Burton, 1991, with permission). Scale bar, 1 mm. $A$, Anterior; $M$, medial; $L$, lateral.
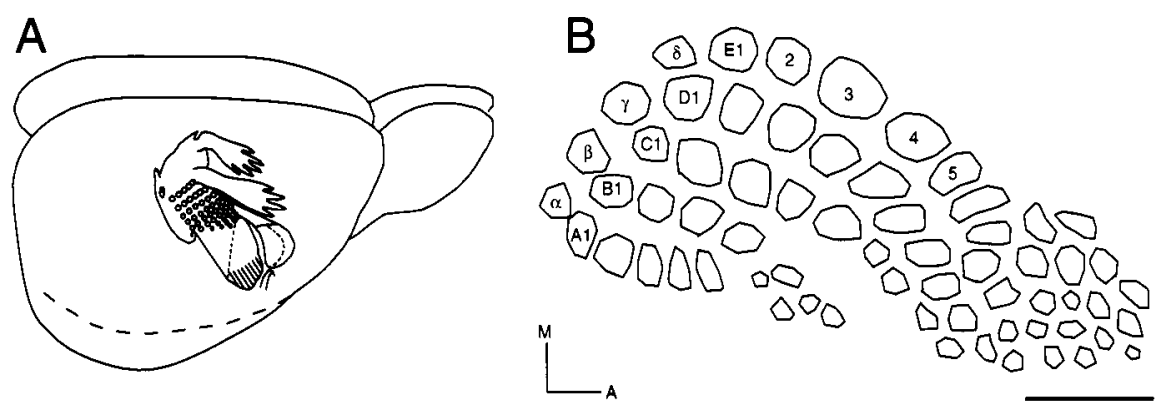

The animals were anesthetized with intramuscular administration of ketamine $(20 \mathrm{mg} / \mathrm{kg})$ and xylazine $(6 \mathrm{mg} / \mathrm{kg})$. Each animal received atropine sulfate $(0.05 \mathrm{mg} / \mathrm{kg})$ to reduce bronchial secretions and chloromycetin sodium succinate $(50 \mathrm{mg} / \mathrm{kg})$ to prevent infection. The animals were intubated with a 16 gauge plastic tube before being immobilized in a stereotaxic frame. Perioperative anesthesia was maintained by ventilation with a 2:1 mixture of nitrous oxide and oxygen containing $0.5 \%$ halothane. End tidal $\mathrm{CO}_{2}$, cardiac rate, and body temperature were monitored throughout the procedure. The body temperature was maintained with a thermostatically controlled homeothermic blanket. Corneal drying was prevented with ophthalmic ointment. Two small holes were made in the cranium overlying the right SI whisker representations as reported previously (Alloway et al., 1999). A carbon fiber electrode (Armstrong-James and Millar, 1979) was inserted through each cranial opening and lowered to a cortical depth of 800-1000 $\mu \mathrm{m}$. Extracellular recordings of multiunit activity were amplified and displayed by conventional means, and individual whiskers were stimulated with a slender rod to determine the best whisker for activating neuronal discharges.

Tracer injections and histochemistry. The two electrophysiologically identified whisker representation sites received injections of either $10 \%$ biotinylated dextran amine (BDA) (Molecular Probes, Eugene, OR), or 10\% rhodamine-conjugated dextran amine [FluoroRuby (FR), Molecular Probes]. FR was loaded into a glass micropipette $(100 \mu \mathrm{m}$ tip) cemented to a $1.0 \mu$ l Hamilton syringe that was placed in a microinjection unit (Kopf 5000, Kopf Instruments, Tujunga, CA). The pipette was inserted perpendicular to the cortical surface, and a volume of $25 \mathrm{nl}$ of FR was injected at a depth of $1400 \mu \mathrm{m}$ below the pial surface. After $5 \mathrm{~min}$, the pipette was retracted to $1200 \mu \mathrm{m}$, and $25 \mathrm{nl}$ of FR was deposited. Five minutes later a third $25 \mathrm{nl}$ volume of FR was injected at a depth of $1000 \mu \mathrm{m}$. To avoid backflow of the tracer, the pipette was left in position for $10 \mathrm{~min}$ before retraction. BDA was injected iontophoretically through a glass micropipette $(35-40 \mu \mathrm{m}$ tip). Three injections were placed at cortical depths of 1400,1200 , and $1000 \mu \mathrm{m}$, respectively, by passing a pulsed $4.0 \mu \mathrm{A}$ current (duty cycle $7 \mathrm{sec}$ ) through the pipette for 7-8 $\mathrm{min}$.

After a survival period of 1 week, each rat was reanesthetized with an intraperitoneal injection of sodium pentobarbital $(50 \mathrm{mg} / \mathrm{kg})$ and perfused transcardially with cold saline containing $1000 \mathrm{U}$ heparin and $20 \mathrm{mg}$ lidocaine, followed by $500 \mathrm{ml}$ cold $4 \%$ paraformaldehyde in $0.1 \mathrm{M}$ phosphate buffer, $\mathrm{pH} 7.4$, and finally another $500 \mathrm{ml}$ of the same solution with $5 \%$ sucrose added. The brains were removed and cryoprotected in $4 \%$

\begin{tabular}{lllll}
\hline \multicolumn{4}{l}{ Table 1. Location, size, and relation between BDA and FR injection sites } \\
Case \# & BDA & FR & $\begin{array}{l}\text { Separation } \\
(\mu \mathrm{m})\end{array}$ & $\begin{array}{l}\text { Total } \\
\text { overlap }(\%)\end{array}$ \\
\hline Within rows & & & & \\
D44 & C2, D2 & D4, 5 & 730 & 13.4 \\
D45 & E4 & E2 & 760 & 20.0 \\
D46 & E5 & E2, 3 & 930 & 12.1 \\
D53 & ABS & E2 & 1700 & 1.7 \\
D43 & $\delta, \gamma$ & D4 & 1740 & 8.7 \\
Across rows & & & & \\
D48 & D2 & C3 & 120 & 14.5 \\
D51 & C2, 3 & E2 & 630 & 9.6 \\
D42 & C4, 5 & E1, 2 & 850 & 1.3 \\
D41 & C4 & E2 & 880 & 1.8 \\
D52 & B3, 4 & E2 & 1200 & 1.7 \\
D40 & E2 & C4, C5 & 1370 & 2.7 \\
D49 & B2, 3 & E3 & 1530 & 0.5 \\
D55 & A6, B6 & ABS & 2000 & 4.4
\end{tabular}

ABS, Anterolateral barrel subfield. paraformaldehyde with $30 \%$ sucrose. Subsequently the right cerebral cortex was dissected out as a slab and flattened between two glass slides. Tangential sections of the cortical slab and transverse sections of the brainstem were cut at $50 \mu \mathrm{m}$ on a freezing microtome.

Alternate serial sections from the flattened cortex were processed for BDA as described previously (Kincaid and Wilson, 1996; Alloway et al., 1998 , 1999). The remaining alternate sections through SI cortex were processed for cytochrome oxidase $(\mathrm{CO})$ using a previously described procedure (Wong-Riley, 1979; Land and Simons, 1985; Alloway et al., 1999). The complete series of transverse sections through the brainstem was processed for BDA according to steps 1-7 in Lanciego and Wouterlood (1994) using a streptavidin-biotinylated horseradish-peroxidase complex (Amersham, Buckinghamshire, UK) as a substitute for the avidinbiotin solution used in the original protocols. Sections were mounted on gelatin-coated glass and coverslipped with Eukit.

Digitization, 3-D reconstruction, and data analysis. The distribution of labeled axons within the pontine nuclei and several anatomic landmarks were recorded using an image-combining computerized microscope system, based on a Leica DMR microscope. Details on software and technical solutions are reported in Leergaard and Bjaalie (1995). Complete series of sections through the pontine nuclei were digitized using a Leitz Plan Fluotar $25 \times$ or $40 \times$ lens. The ventral surface of the pons, the outlines of the pontine gray, the contours of the corticobulbar and corticospinal fiber tracts (in the following referred to as the peduncle), the midline, and the fourth ventricle served as reference lines.

The plexuses of BDA-labeled axons were viewed with translucent light, and the FR-labeled axons were viewed with excitation light of 515-560 nm (Leitz N2.1 filter block). The labeled plexuses within the pontine gray were coded semiquantitatively as points (see also Leergaard and Bjaalie, 1995; Leergaard et al., 1995, 2000). In areas with a low density of labeling, point coordinates were placed at regular intervals along the length of single axons. In areas with dense labeling it was impossible to assign coordinates to individual fibers. Although the distribution of labeling was recorded accurately, a rough correspondence was sought between the density of labeling and number of digitized points, resulting in tight point clusters corresponding to dense axonal plexuses. The two categories of labeling were digitized independently, and the correspondence of labeling and digitized image for both labeled categories was studied carefully using alternating light microscopy and fluorescence microscopy with the $25 \times$ and $40 \times$ lenses.

For the 3-D visualization and analysis of the distribution of labeling, we used program Micro3D for Silicon Graphics workstations (Oslo Research Park, Oslo, Norway), which was developed at the Neural Systems and Graphics Computing Laboratory, University of Oslo (http://www.nesys. uio.no/). Forerunners of this software were used in several recent investigations (Leergaard et al., 1995, 2000; Malmierca et al., 1995, 1998; Bjaalie et al., 1997a,b; Berg et al., 1998; Bajo et al., 1999; Vassbø et al., 1999). The digitized sections were aligned interactively on the screen with the aid of the reference lines (see above). Each section was assigned a $z$-value defined by its thickness and serial number. Tissue shrinkage was estimated by comparison of drawings of sections before and after the histochemical processing. To maintain correct in vivo proportions in the 3-D reconstructions, section thickness (which was originally $50 \mu \mathrm{m}$ ) was set to $47.5 \mu \mathrm{m}$ as an adjustment for a linear shrinkage of $\sim 5 \%$. To facilitate the alignment, real-time rotation with inspection of the 3-D reconstructions from different angles of view was used. The reconstructions served as a basis for the further visualization and analyses of densities and distribution of point clusters. Surface modeling was performed with a simple triangulation method or with the use of the software library SISL (SINTEF Spline Library) (cf. Bjaalie et al., 1997a).

To facilitate the visualization of results and comparison among cases, we used the standard pontine coordinate system and related diagrams for visualization presented in Leergaard et al. (2000). This coordinate system is divided into relative values from 0 to $100 \%$. The origin of coordinates is defined by the intersection of the midline and a line perpendicular to the midline and tangential to the rostral border of the pontine nuclei. Only the two halfway $(50 \%)$ reference lines are shown in the figures.

The degree of overlap between the BDA- and FR-labeled terminal fields was estimated by subdividing individual sections into an array of $35 \mu \mathrm{m}^{2}$ bins and counting the numbers of digitized coordinate pairs per bin. Similar solutions were used previously by others (He et al., 1993; Alloway et al., 1999). To avoid analyzing areas with very low density of labeling, 

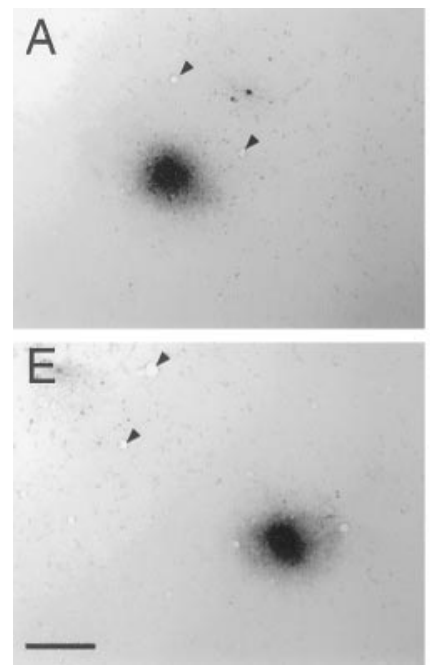
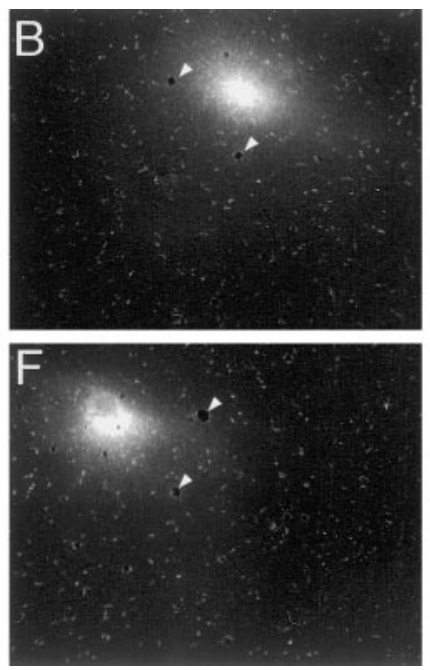
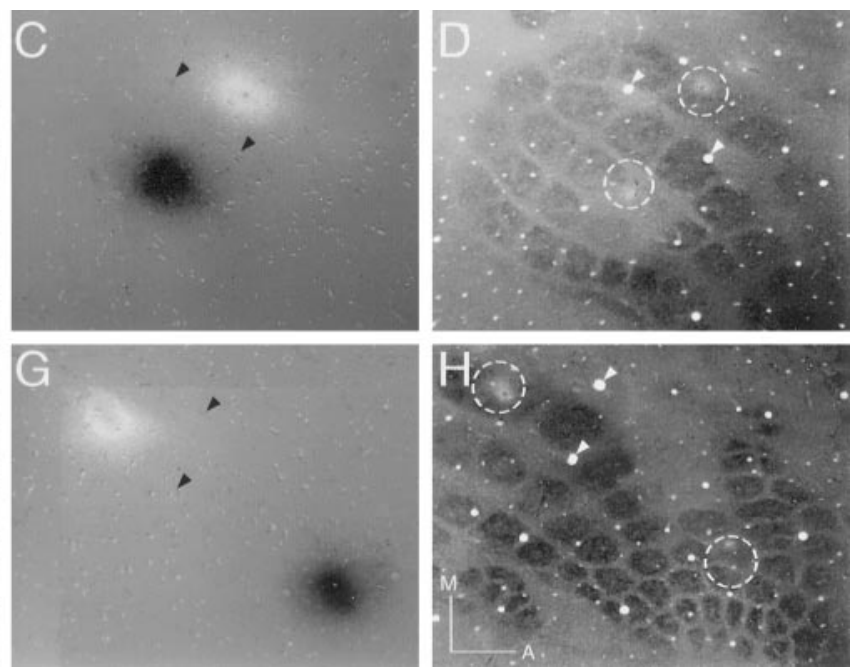

Figure 2. Topography of BDA and FR injections in two representative cases ( $A-D$ : D51, $E-H$ : D53). $A, B$ and $E, F$ are corresponding photomicrographs of tangential sections through layer V of SI, revealing the extent of the BDA and FR injection sites in cases D51 and D53, respectively. $C$ and $G$ are overlays of the photomicrographs in $A, B$ and $E, F$, respectively. $D$ and $H$ show tangential sections (corresponding to $A-C$ and $E-G$, respectively) through layer IV of SI cortex labeled for CO to indicate the location of individual whisker barrels. Dashed circles indicate the boundaries of the injection sites. Arrowheads indicate corresponding sets of blood vessels in each panel. In case D51 $(D)$, the BDA injection is located between barrels C2 and C3, whereas the FR injection is restricted to E2. In case D53 $(H)$, the BDA injection site is located in the anterolateral barrel subfield, and the FR injection is located in E2. Scale bar, $500 \mu \mathrm{m}$. $A$, Rostral; $M$, medial.

bins containing only one point were excluded. Because areas with high density of labeling were recorded by approximation, no attempt was made to differentiate the labeled zones according to density. Bins containing two or more red or blue points were defined as "red" or "blue," respectively. Bins containing two or more points of both categories were defined as "white" bins, representing overlap. The numbers of red, blue, and white bins were counted for each section, summed across sections, and used to estimate an index of total overlap. The overlap estimate is highly influenced by bin size and threshold criteria. If small bins (e.g., $\left.5 \mu \mathrm{m}^{2}\right)$ and high thresholds (e.g., $>10$ points per bin) were used, the amount of overlap appeared minimal, whereas by comparison, large bins and low thresholds resulted in increased overlap. We chose the same bin size $\left(35 \mu \mathrm{m}^{2}\right)$ as used by Alloway et al. (1999), partly to facilitate comparison across brain regions and partly because similar arguments for this bin size were applicable to the pontine nuclei. Bins $>35 \mu \mathrm{m}$ tended to yield overlap in situations where the labeled terminals appeared segregated. Smaller bins were not used because clearly overlapping areas then tended to be defined as segregated.

We have based our analysis on an overlap index defined as the number of white bins divided by the number of red, blue, and white bins. Statistical ANOVA was performed using the Statistical Package for the Social Sciences (SPSS Inc., Chicago, IL).

Illustrations were assembled with Showcase (Silicon Graphics, Mountain View, CA), Adobe Illustrator 8.0, and Adobe Photoshop 5.0. Digital photomicrographs were obtained through a CoolSnap camera (Photometrics, Tucson, AZ). The gray-scale levels of the images were optimized using the "curves" function for "autolevels" in Adobe Photoshop.

\section{RESULTS}

For this report, results from four representative animals are illustrated and described in detail. More documentation, including original data sets with 3-D spatial coordinates, is available at http://www.nesys.uio.no/ and http://www.cerebellum.org/.

\section{Injection sites}

In each experiment, restricted injections of BDA and FR were placed into separate regions of the SI barrel cortex (Fig. 1). Ten of the 13 experiments were selected by criterion of labeling in the neostriatum and thalamus and were used previously in a study of corticostriatal organization (Alloway et al., 1999). All cases contained anterograde BDA and FR labeling in the pontine nuclei. All injections were restricted to the gray matter. The outlines and position of the injection sites were estimated with respect to the whisker barrels by comparison of adjacent BDA- and COprocessed tangential sections through cortical layers IV and V. The position of injections in the whisker barrel field corresponded to the electrophysiological recordings made before tracer injection. The diameter of the injection sites (Table 1), defined by the maximum width of dense staining in layer $\mathrm{V}$ (the cortical layer containing cell bodies of corticopontine neurons), ranged from 200 to $800 \mu \mathrm{m}$. Each injection involved one to two barrels (Table 1). In five cases, both tracers were confined to the same row of barrels ("within rows"), and in eight cases the tracers were injected into separate rows of barrels ("across rows"). The edge-to-edge separation between the two injection sites was measured in layer V (Fig.
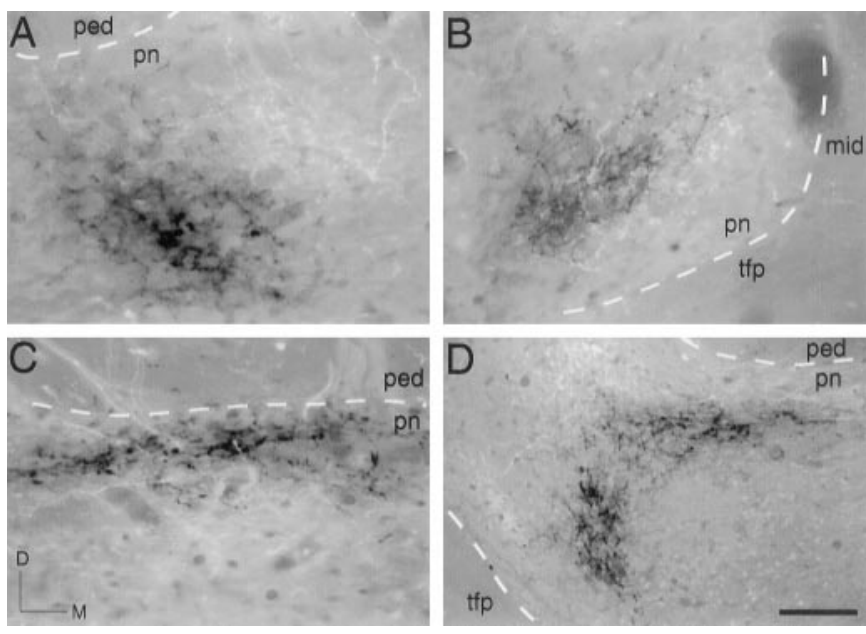

Figure 3. Photomicrographs of FR and BDA labeling in transverse sections through the pontine nuclei. $A, C$, Case D44; $B$, case D45; $D$, case D46. Each image is an overlay of two transparent photomicrographs, viewed by fluorescence microscopy and conventional light microscopy for visualization of FR and BDA, respectively. $A$ shows partly overlapping clusters of FR and BDA fibers located slightly rostral to the midpontine level. $B$ shows partly overlapping FR and BDA clusters located close to the midline at the midpontine level. $C$ shows elongated, partially overlapping FR and BDA clusters along the ventral aspect of the peduncle. Compared with the FR labeling, the BDA labeling is slightly shifted toward dorsal and thus is considered to be located more "externally." $D$ shows closely associated, curved FR- and BDA-labeled clusters located laterally in the pontine nuclei. The clusters are incomplete parts of a lamellar subspace. The FR labeling is shifted slightly toward dorsal and lateral and surrounds the BDA labeling externally. Further documentation of FR and BDA images is available at http://www.nesys.uio.no/and http://www.cerebellum.org/. Scale bar: $A-C, 50 \mu \mathrm{m} ; D, 100 \mu \mathrm{m}$. $D$, Dorsal; $M$, medial; mid, midline; ped, peduncle; $p n$, pontine nuclei; tfp, transverse fibers of the pons. 
A
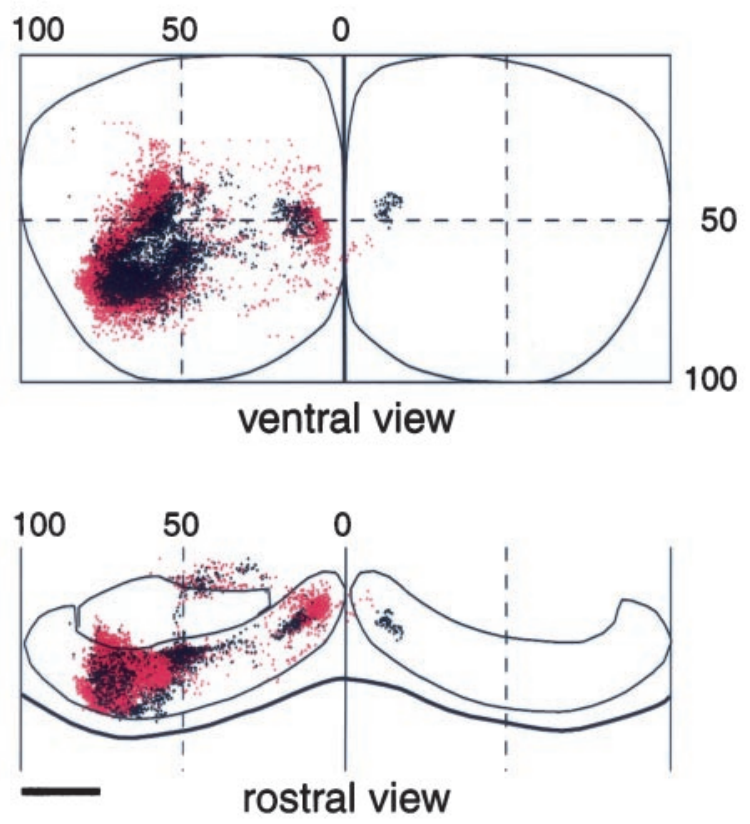

C
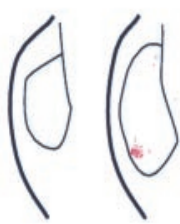

100 90

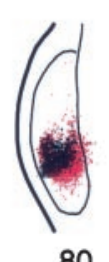

80

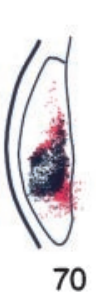

70
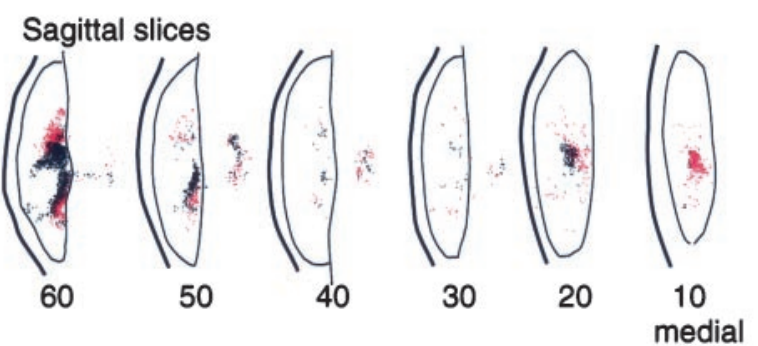

B
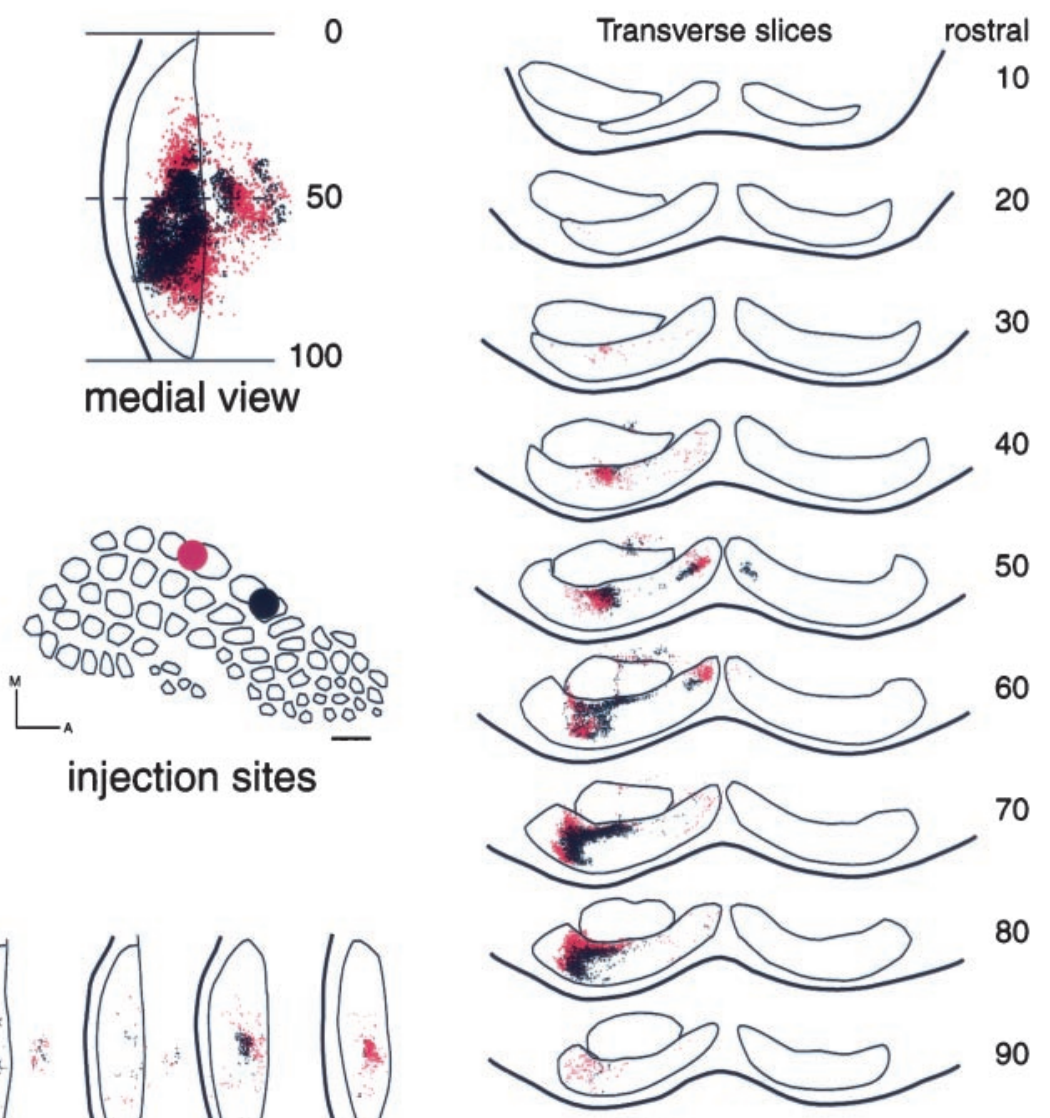

90

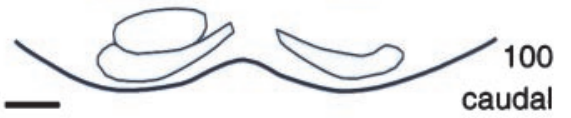

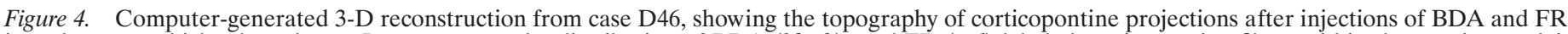

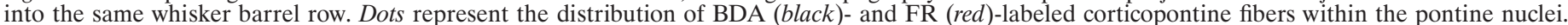

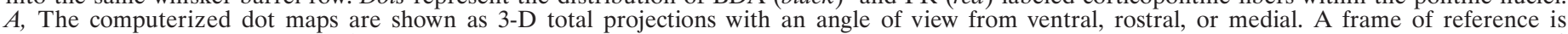

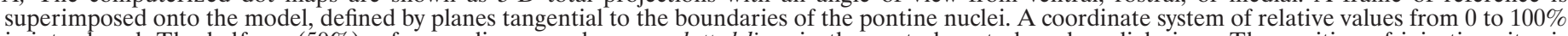

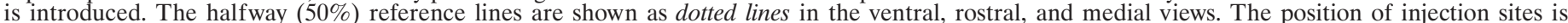

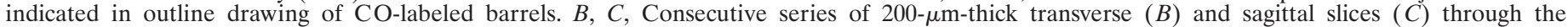

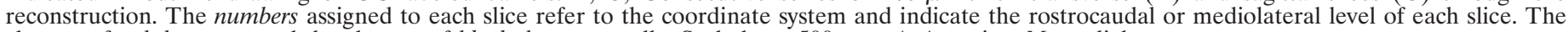
clusters of red dots surround the clusters of black dots externally. Scale bars, $500 \mu \mathrm{m}$. $A$, Anterior; $M$, medial.

2). Injection sites never overlapped. Photomicrographs of representative injection sites are shown in Figure 2.

\section{General features of labeling and overall topography}

The BDA and FR injections gave rise to labeling in several intracortical and subcortical targets. Topographically organized fiber complexes were found in the ipsilateral neostriatum and in the thalamic nuclei together with retrogradely labeled cells (Alloway et al., 1999). Within the ipsilateral pontine nuclei, anterogradely labeled fibers branched extensively to form several dense axonal plexuses that were assumed to represent terminal fields (Fig. 3). We observed beaded varicosities along the trajectories of all BDA and FR fibers inside the pontine nuclei. Recent ultrastructural analyses demonstrated that similar varicosities along corticostriatal fibers contained synaptic vesicles and thus may represent corticostriatal synapses (Kincaid et al., 1998). Because the beaded varicosities observed in the pontine nuclei may represent synaptic contacts (Mihailoff et al., 1981), we have chosen to record the complete trajectory of the labeled fibers within the pontine nuclei.

The shape, size, and density of labeled plexuses produced by the two tracers were very similar. Viewed in single sections, the BDA and FR labeling were aggregated in sharply defined, rounded patches that were 50-200 $\mu \mathrm{m}$ in diameter (Fig. 3A,B) and elongated thin bands that were $\sim 50-100 \mu \mathrm{m}$ wide (Fig. $3 C$ ). The computerized reconstructions showed that the two-dimensional (2-D) patches are parts of 3-D clusters (Figs. 4, 5). The shape and distribution of the clusters suggest that they are incomplete components of concentric layers or lamellae (Fig. 6).

In general, the aggregates of BDA and FR labeling were colocalized and frequently partially overlapping (Fig. 3). Completely segregated patches of BDA and FR labeling were often observed, whereas completely overlapping patches were not found. The widespread distribution and lamellar shape provides a large side-by-side interface between neighboring terminal fields. Furthermore, after injection of BDA and FR into different SI whisker barrels, we often observed clusters of BDA- and FR-labeled fibers laterally, medially, and dorsally in the pontine nuclei (Figs. 4-6), which indicate the presence of multiple pontine body representations. This is in agreement with previous findings from the mapping of larger SI body representations onto the pontine nuclei (Leergaard et al., 2000).

To describe the spatial relationships between labeled clusters, we defined the internal region of the pontine nuclei as a centrally 


\section{Across rows}

A
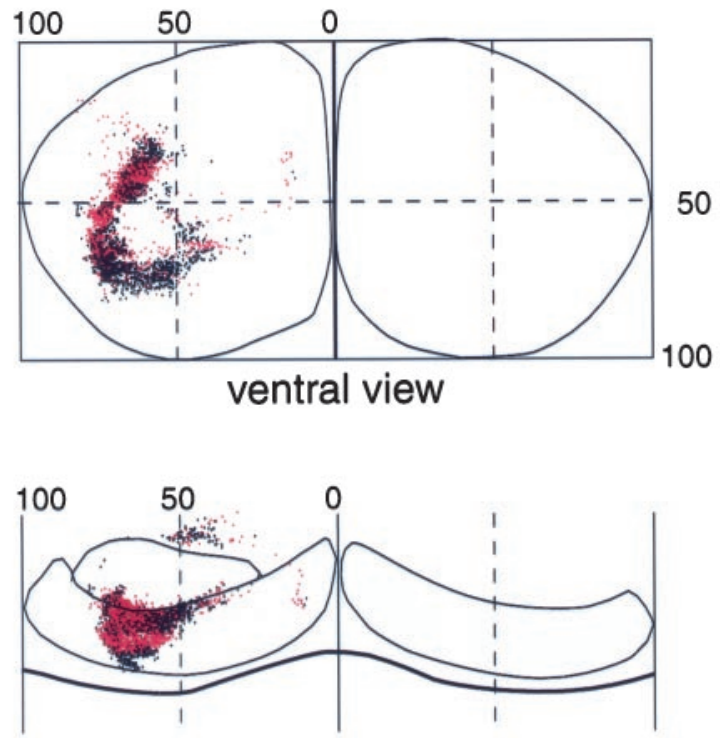

rostral view

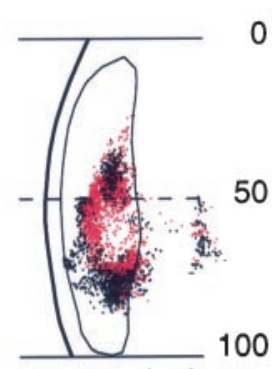

medial view

B
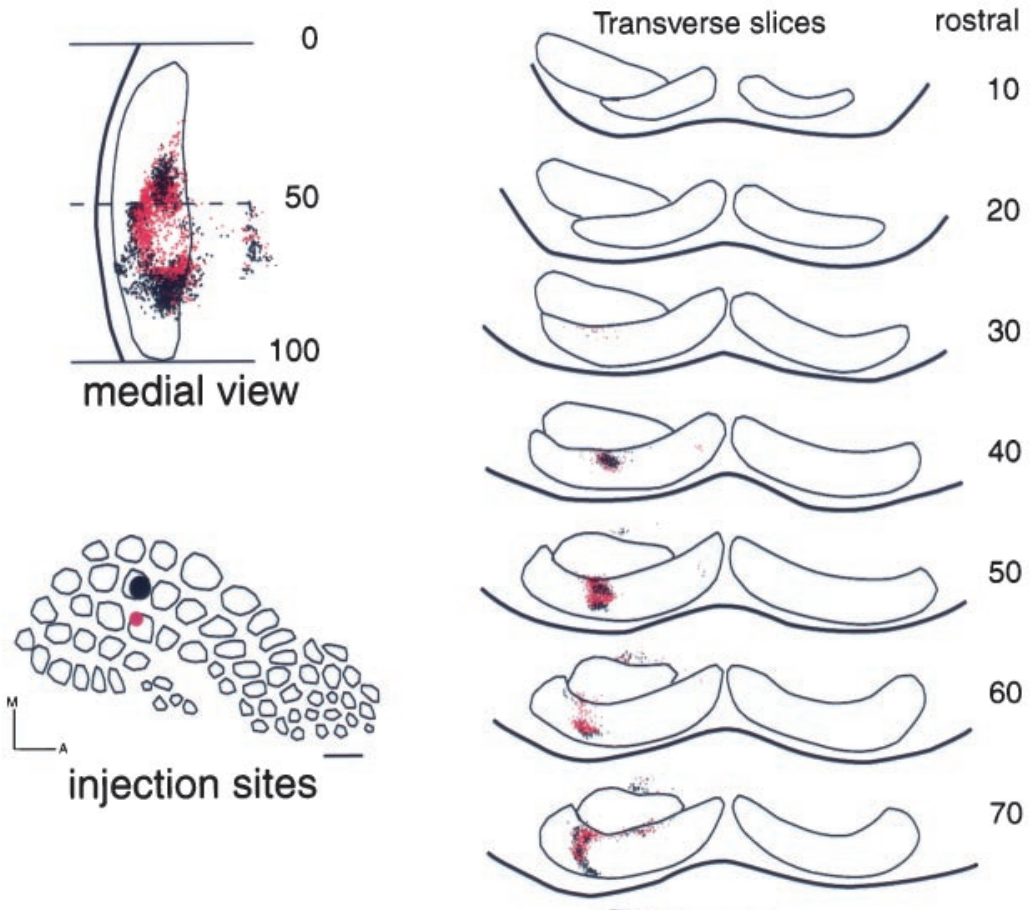

C

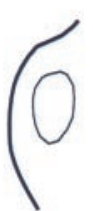

100 lateral
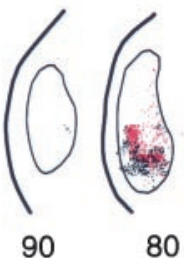

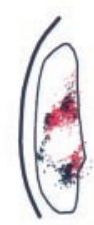

70

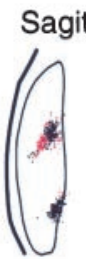

60

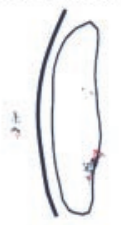

50

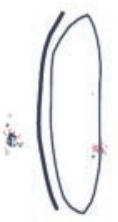

40

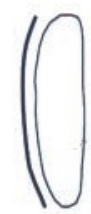

30

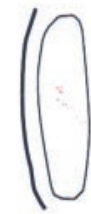

20

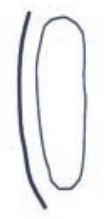

10

medial

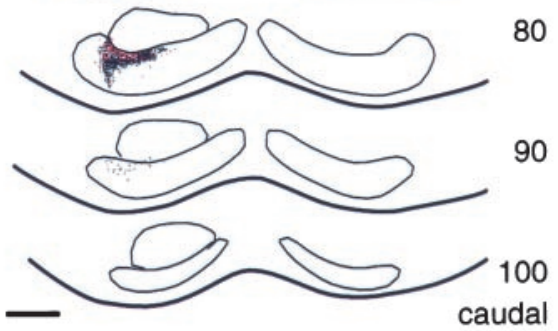

caudal

Figure 5. Computer-generated 3-D reconstruction from case D48, showing the topography of corticopontine projections after injections of BDA and FR into different whisker barrel rows. Presentation as in Figure 4. The clusters of red and black dots are located within a single narrow lamella-shaped volume, with a dorsal (FR) to ventral (BDA) shift in the preponderance of labeling. Scale bars, $500 \mu \mathrm{m}$. $A$, Anterior; $M$, medial.

located core, relatively close to the peduncle. Regions outside this core, that is, medial, lateral, ventral, dorsal, caudal, or rostral to it, were referred to as external (see also Leergaard et al., 1995, 2000). There was little variation among cases in the 3-D shape of labeling from different whisker barrels. In all cases, each injection produced ipsilateral labeling in several clusters. The largest cluster was located laterally and caudally, relative to the central core of the pontine nuclei (Figs. $4 A$, ventral view, $B, 6 A, C$ ). A smaller cluster was located close to the midline (Figs. $4 A, 5 B$, ventral view, rostral view, $6 A$ ). These findings are consistent with previous work indicating the presence of medial and lateral pontine terminal fields after tracer injections into the SI facial and vibrissal representations (Wiesendanger and Wiesendanger, 1982; Mihailoff et al., 1985; Lee and Mihailoff, 1990; Panto et al., 1995). In addition, we frequently observed loose axonal plexuses along the dorsal aspect of the ipsilateral peduncle and in the contralateral pontine nuclei (Figs. 4A, rostral view, $5 A$, rostral view).

We have previously mapped the pontine projections from the major SI-body representations using a single-tracing approach (Leergaard et al., 2000). When compared with those previous results, it appears that the SI whisker representations surround the perioral projection area, which is located centrally in the pontine nuclei. The whisker representations are thus intercalated between the perioral representations and the more externally located projections from the trunk and neck [compare Figs. 4 and 5 with Leergaard et al. (2000), their Figs. 8 and 9). Hence, the present results are consistent with our previously published 3-D map of the corticopontine projections from SI.

\section{Corticopontine projections from SI barrels in the same row}

After BDA and FR injections into the same row of barrels, the ensuing BDA and FR labeling was distributed in a pair of concentrically arranged lamellae. Figures 4 and 6 show that the FRlabeled clusters in case D46 occupy more lateral, medial, and dorsal locations than the corresponding BDA clusters. Thus, the FR clusters in this case, representing the more caudal whisker, surround the BDA clusters externally. The internal to external shift in location of labeling is shown to advantage in the transverse and sagittal slices through the reconstruction (Fig. 4B, $C$, slices 50-80).

The BDA and FR clusters in case D46 were partially overlapping at practically all rostrocaudal or mediolateral levels containing labeling (Figs. 3D, 4, 7A,B). The total amount of overlap in this case was $12.1 \%$. The separation of the two injections was $930 \mu \mathrm{m}$. In another within row experiment (D53), the separation distance between the injection sites was much larger $(1700 \mu \mathrm{m})$. Although the same overall shape and lamellar distribution pattern was seen in this case, the labeled clusters were clearly more segregated (Fig. $7 C, D)$, and the amount of total corticopontine overlap was only $1.7 \%$. Five within row experiments demonstrated decreasing amount of overlap with increasing separation of the cortical sites of origin (Table 1, Fig. 8). 


\section{Within rows}

A
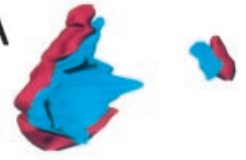

B
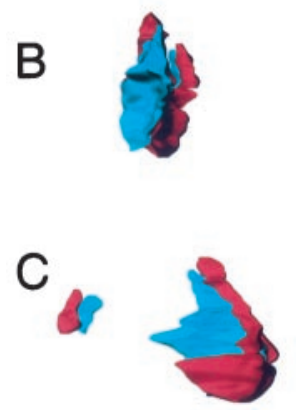
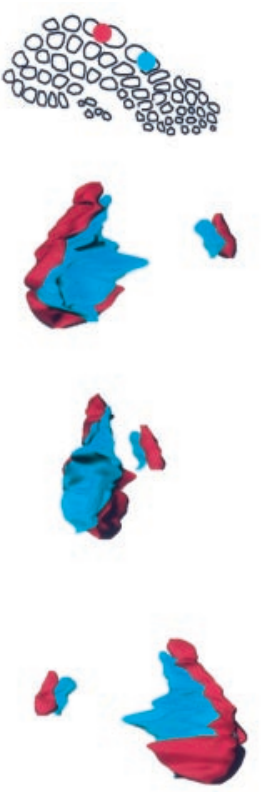
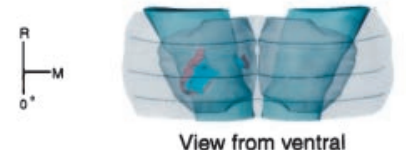

$\downarrow_{90}$

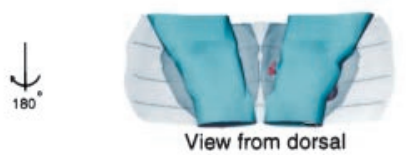

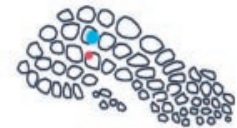

Across rows
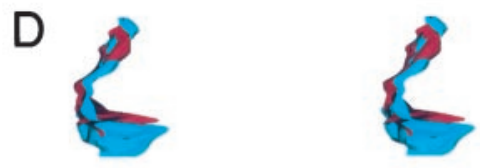

$E$
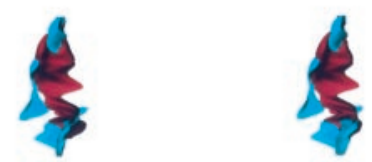

$\mathrm{F}$

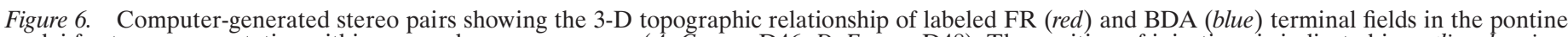

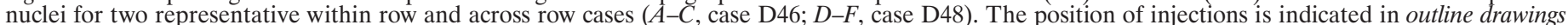

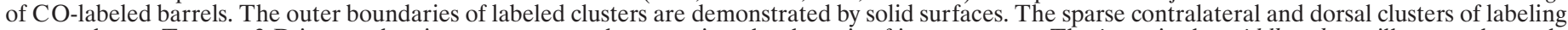

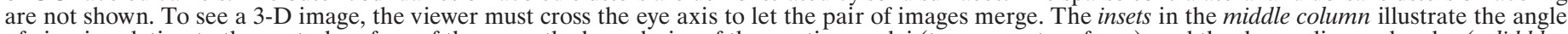

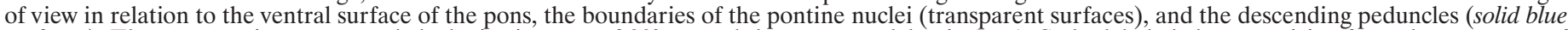

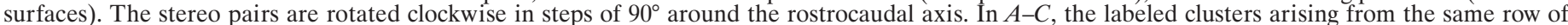

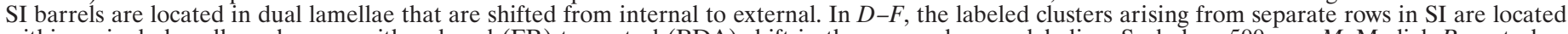

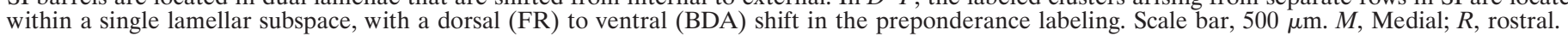

\section{Corticopontine projections from SI barrels in different rows}

When BDA and FR injections were placed in different rows, the ensuing labeled terminal fields had a lamellar shape as described above. Instead of producing a dual lamellar pattern, however, the labeled clusters for both tracers appeared to be localized within a single lamellar volume. Case D48 illustrates this finding in both sagittal and transverse slices (Fig. 5B, slices 50-80; $5 C$, slices $60-80$ ). The BDA labeling arising from a barrel in row $\mathrm{D}$ tended to extend more ventrally in the pontine nuclei than the FR labeling arising in an adjacent barrel in row C (Figs. 5B, slices 60-80, $6 D-F)$. These topographic differences however, were less conspicuous than the marked inside-out pattern observed in the within row cases. In case D48, the injection sites were separated by only 120 $\mu \mathrm{m}$ in cortical layer $\mathrm{V}$, and total corticopontine overlap was $14.5 \%$ (Fig. $7 E, F)$. In another across row case (D51), the two injection sites were separated by $630 \mu \mathrm{m}$ (Fig. 7G,H). The labeled clusters in D51 were also confined within a lamellar subspace. The clusters appeared to be more segregated than in case D48, as indicated by a reduction in the total amount of corticopontine overlap (9.6\%). As Table 1 indicates, we observed decreasing amounts of corticopontine overlap with increasing separation of the tracer injection sites (Table 1, Fig. 8).

\section{Quantitative estimate of overlap}

The degree of overlap between the BDA- and FR-labeled terminal fields was calculated by subdividing individual sections into an array of $35 \mu \mathrm{m}^{2}$ bins and counting the numbers of digitized coordinate pairs per bin (for details, see Materials and Methods). The total overlap index ranged from 0.5 to $20 \%$, with a mean index of overlap of $7.1 \pm 1.8 \%$ (SEM). Generally, the index of overlap appeared higher in the within row than in the across row group (Table 1, Fig. 8). The highest overlap (20\%) was observed after nearby injections in the same row of whiskers (Table 1, case D45). The lowest overlap $(0.5 \%)$ was found in a case with injections in separate rows (Table 1, case D49). Furthermore, the amount of overlap varied relative to the separation of injection sites (Table 1, Fig. 8). To compare the effect of these two parameters, the within row and across row groups were sorted into groups with $<1000 \mu \mathrm{m}$ separation ("closer separation") and groups with injection sites separated by $1000 \mu \mathrm{m}$ or more ("farther separation"). For these four groups, we wanted to describe the variation of overlap as a function of two categorical parameters: the relative position and the separation of injections. A two-way ANOVA showed that there was no interaction between the two parameters $(p=0.898)$. Thus, the difference between the groups, with respect to one parameter, is the same, regardless of the level of the other factor. Furthermore, a two-way ANOVA (without interaction) showed that there was a significant reduction in overlap from the within rows to the across rows group $(p=0.049)$ and a significant reduction from the closer separation to the farther separation groups $(p=0.031)$. Based on the ANOVA model, the reduction in average overlap was $43.5 \%$ from the within rows to the across rows group and $47.4 \%$ from the closer separation to the farther separation group. In summary, our analyses show that the degree of partial overlap between pontine terminal fields decreases with increasing separation of the cortical sites of origin and that there is a significantly higher degree of overlap between within row cases as compared with across row cases (see also Fig. 8).

\section{Comparison of corticopontine and corticostriatal projections}

Ten animals used in the present study were previously used in a comparable study of the neostriatum (Alloway et al., 1999). Four of these animals were within row cases (D43, D44, D45, D46) and six were across row (D41, D42, D48, D49, D51, D52). Comparisons of these two brain regions revealed that the corticopontine projections have several principles of organization in common with corticostriatal projections. Thus, in both target regions, terminal fields were somatotopically organized in distinct, partially overlapping clusters that reside in curved lamellar zones (Fig. 9). As shown in the present study, corticopontine projections from the same row of barrels were distributed side by side in adjacent lamellae, whereas projections originating in different rows occupied largely separate regions within a single lamellar volume (Fig. 9G-I). In the corticostriatal projection, 

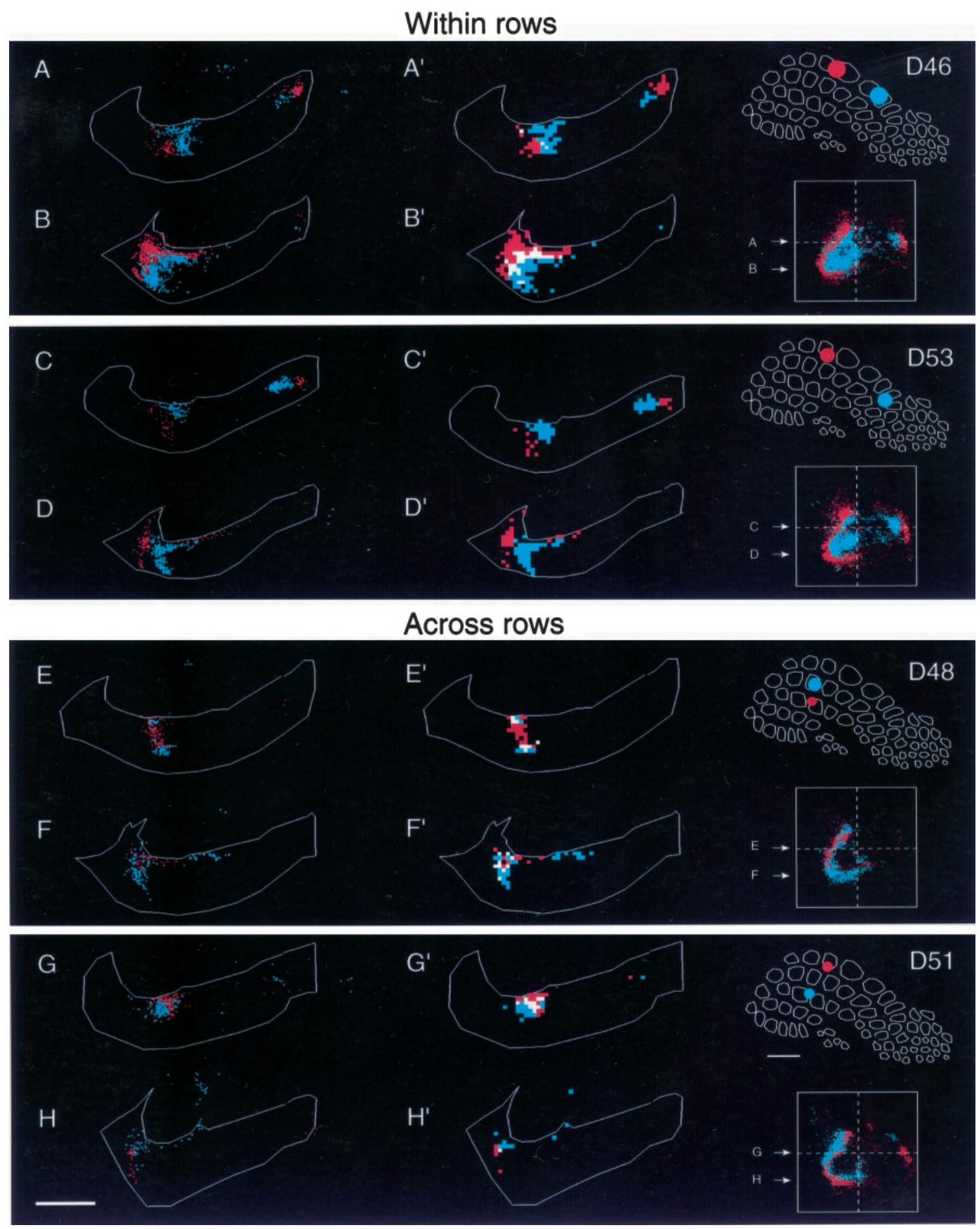

Figure 7. Dot maps and overlap analysis of selected transverse sections from four representative cases $(A, B$ : case D46, $C, D:$ case D53, $E, F$ : case D48, $G, H$ : case D51). Sections are selected from rostrocaudal levels $50 \%(A, C, E, G)$ and $80 \%(B, D, F, H)$, as indicated by arrows in the insets. The insets in the right column show the BDA (blue) and FR (red) injection sites and corresponding 3-D reconstructions in a ventral view (see also Figs. 5 and 6 ). $A-H$ show blue and red dots representing the distribution of BDA and FR labeling within the pontine nuclei, whereas $A^{\prime}-H^{\prime}$ show an overlap analysis of the same sections. Each digitized dot map is subdivided into $35 \mu \mathrm{m}$ bins, and the number of BDA and FR dots is counted in each bin. Bins containing at least two BDA or FR dots are colored blue or red, respectively; those containing at least two of each type are colored white (cf. Materials and Methods). Projections from separated whisker barrels partly overlap in the pontine nuclei. The amount of overlap (Table 1) decreases when the distance separating the injection sites increases (compare $A^{\prime}, B^{\prime}$ with $C^{\prime}, D^{\prime}$, and $\mathrm{E}^{\prime}, F^{\prime}$ with $\left.G^{\prime}, H^{\prime}\right)$. Inset frames are $2 \times 2 \mathrm{~mm}$. Scale bars, $500 \mu \mathrm{m}$.

however, projections originating in different whisker barrel rows terminated in different lamellae (Fig. 9E) (Brown et al., 1998; Alloway et al., 1999; Wright et al., 1999). In further contrast to the corticopontine projections, corticostriatal projections originating from cortical sites in the same SI barrel row terminated in lamellae with ends that merged into each other to occupy a single, more elongated lamellar volume (Fig. 9) [see also Alloway et al. (1999), their Fig. 10]. Despite differences in the anatomic organization of the corticopontine and corticostriatal projections, both pathways displayed a significantly higher degree of overlap for projections arising from the same row of barrels (Alloway et al., 1999; present study). 


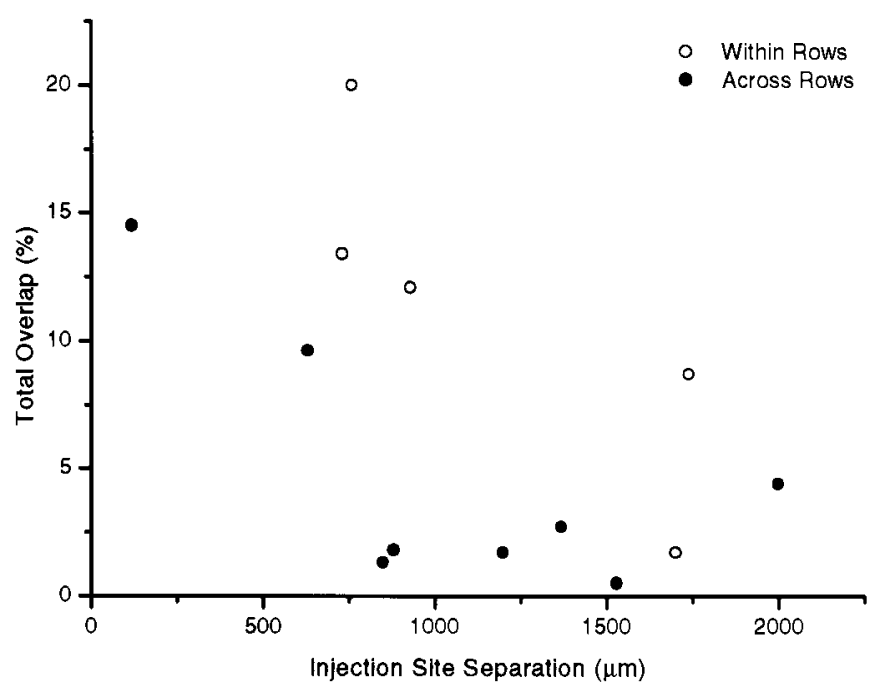

Figure 8. Scatter plot of the variation in overlap of pontine terminal fields as a function of the distance separating injection sites in cortical layer V. The total amount of overlap in the pontine nuclei decreases with increasing separation of the injection sites and is significantly higher among projections originating from barrel columns in the same row of SI cortex (see also Table 1).

\section{DISCUSSION}

This study demonstrates that corticopontine projections from the rodent SI barrel fields are topographically organized. By combining double anterograde tracing with computerized 3-D reconstruction techniques, we found that the projections from individual SI barrels terminate in concentrically arranged lamella-like subspaces.

Clustered projections from barrels in the same row terminate in different lamellae, whereas projections from different barrel rows terminate in regions within the same lamellar subspace. Comparisons of corticopontine and corticostriatal projection patterns in the same animals revealed similarities as well as differences. Although both corticopontine and corticostriatal projections terminate in lamella-shaped regions, barrels within a whisker row project to lamellae organized side by side in the pontine nuclei, but end to end in the neostriatum (Fig. 9). Nevertheless, for both corticof ugal systems, terminal overlap was greater for projections arising from SI whisker barrels in the same row than for projections arising from barrels in different rows. This organizational scheme may shed new light on corticopontine topographic organization, pontine integration of information originating in nearby cortical sites, and putative roles of the pontine nuclei in cerebrocerebellar communication.

\section{Establishment of corticopontine and corticostriatal topography}

Developmentally, there is a striking resemblance between corticopontine and corticostriatal projections. Thus, the topography of these projections seems to correlate with neurogenetic gradients in cortex (source) and target regions. In the cerebral cortex, the neurogenetic gradient extends from anterolateral toward posterior and medial, in parallel with the rows of barrels in SI (Hicks and D’Amato, 1968; Smart, 1984; Bayer and Altman, 1991; Erzurumlu and Jhaveri, 1992; Bishop et al., 2000). A similar gradient was reported for the development of electrophysiological specificity (McCandlish et al., 1993). Thus, anterolaterally located barrels (high numbers) are developed before the more medially and posteriorly located barrels (low numbers). In the pontine nuclei, the early established region is located in the central core (Altman and Bayer, 1978, 1987), and this region receives projections from the early, established anterolateral cortex (Leergaard et al., 1995; see also Leergaard and Bjaalie, 1998; Leergard et al., 2000). The present findings are in agreement with this distribution principle. Thus, SI barrels with high numbers (early) project to a region close to the central core (early), and projections from barrels with lower numbers (later) wrap around this core. In the neostriatum, SI barrels with high numbers project to the ventrolateral part, and barrels with lower numbers project to successively more dorsomedial regions (Alloway et al., 1999; Wright et al., 1999). Again, this topographic distribution follows the neurogenetic gradient of the neostriatum (Bayer and Altman, 1987). The further shaping of the terminal fields in these pathways is presumably accomplished through a multitude of developmental mechanisms.

\section{Representation of cortical barrels in the pontine nuclei}

In virtually all species, corticopontine projections terminate in several, sharply defined clusters (for review, see Brodal and Bjaalie, 1992, 1997). The present study demonstrates that neighboring cortical injections give rise to paired clusters at several locations in the pontine nuclei. This finding indicates the presence of multiple, somatotopically organized representations of the SI map in the pontine nuclei. This intricate corticopontine topography has been interpreted as a basis for the fractured maps of tactile representations found in the cerebellar hemispheres (Schwarz and Thier, 1995). Indeed, the transformation from a cortical 2-D map to a pontine 3-D map gives opportunities for some new neighboring relationships (Leergaard et al., 2000; see also Leergaard and Bjaalie, 1998; Schwarz and Thier, 1999). However, a more characteristic feature of corticopontine mapping is the preservation of somatotopic order. By means of $3-\mathrm{D}$ reconstruction and single tracing techniques in rat, we demonstrated that neighboring relationships of major body representations are largely preserved in the SI-pontine projections (Leergaard et al., 2000). In the present study, we have shown similar preserved spatial relationships among terminal fields originating in different SI whisker barrels. The tendency for continuous topographic representations is further emphasized by the finding of decreasing overlap of terminal fields with increasing separation of cortical site of origin. We therefore hypothesize that there are gradual shifts in the location of terminal fields, i.e., smooth shifts in the cortical influence on neurons in the pontine nuclei. Thus, a linear shift of neural activity within or across rows of barrels in the cerebral cortex would evoke gradual shifts (inside-out or within lamellae, respectively) of activity in multiple pontine neuronal groups.

\section{Local integration or parallel pathways?}

Organized topography is a prerequisite for parallel processing of information originating in different cortical domains. The present study has demonstrated a clustered and lamellar topography for pontine projections from individual cortical barrels that could serve parallel processing functions. Two findings, however, suggest convergence of afferent signals from several barrels onto single pontine neurons. First, there is partial overlap (up to 20\%) (Table 1) between terminal fields after injections into nearby barrels. Second, the pontine terminal fields originating in individual cortical barrels are generally more narrow than the dendritic trees of pontine neurons, as judged from comparisons of our data with available illustrations of Golgi-stained (Mihailoff et al., 1981) or Lucifer Yellow-injected pontine cells (Schwarz and Thier, 1995). A comparison of Golgi-stained pontine neurons (Mihailoff et al., 1981, their Fig. 46) with the tracer-stained terminal fields in our Figure $3 D$ shows the potential for local integration in the pontine nuclei. Thus, in line with the view presented by Malach (1994) for corticocortical connections, we suggest that sharply defined, clustered terminal fields may facilitate integration of signals and increase diversity of inputs to single neurons [see also discussion by Brodal and Bjaalie (1997), Malmierca et al. (1998); for a discussion of contrasting views, see Schwarz and Thier (1999), Bjaalie and Leergaard (2000), Schwarz and Thier (2000)]. The modular organization of the neostriatum has also been viewed as suitable for specific integrative purposes (Graybiel, 1990; Brown, 1992; Brown et al., 1998; Alloway et al., 1999).

In the context of putative parallel and re-entrant circuits (cerebro-ponto-cerebello-thalamo-cerebral loops) (Middleton and Strick, 2000), our data indicate that there is convergence already at 


\section{Within rows}

A

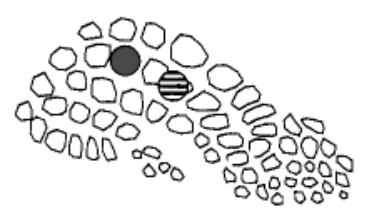

D

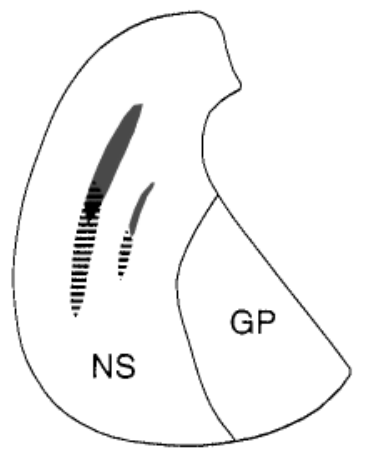

G

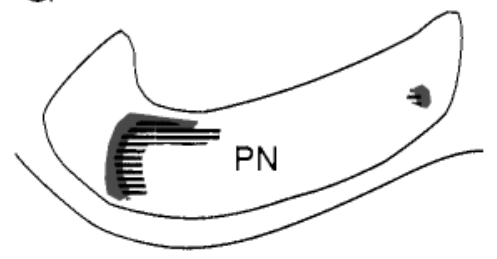

Across rows

B

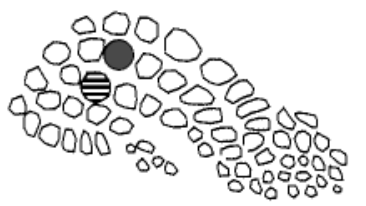

$E$

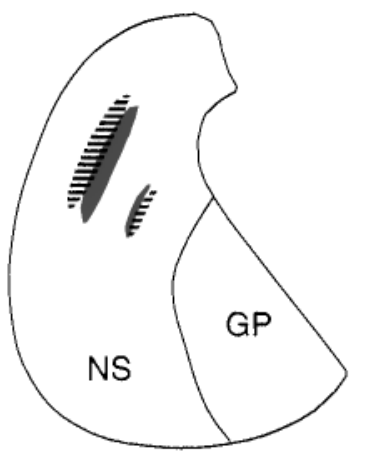

$\mathrm{H}$

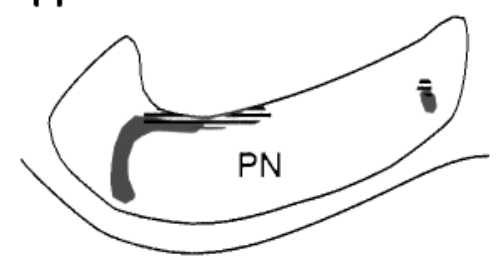

2D topographic layout

C

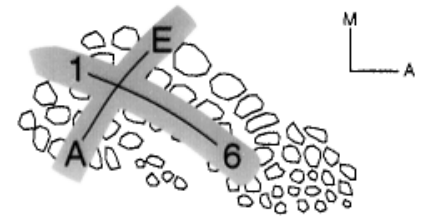

$\mathrm{F}$

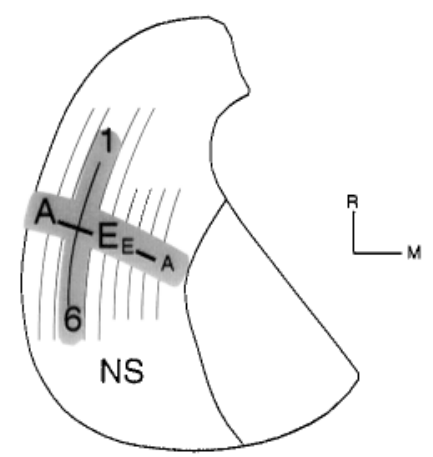

।

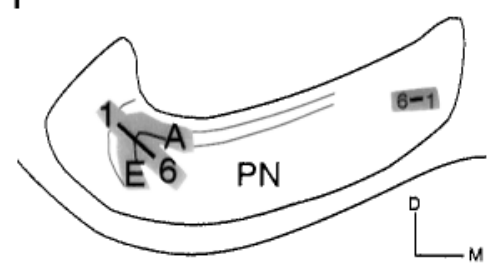

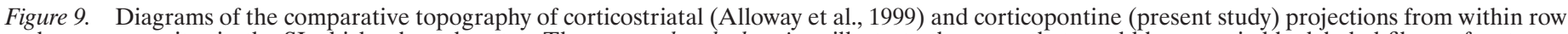

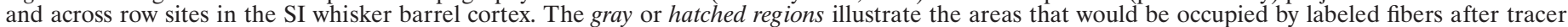

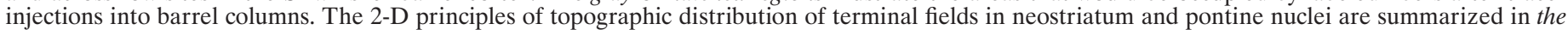

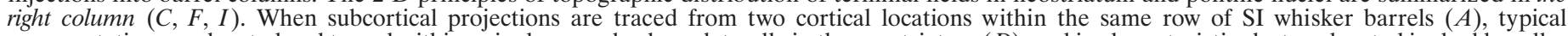

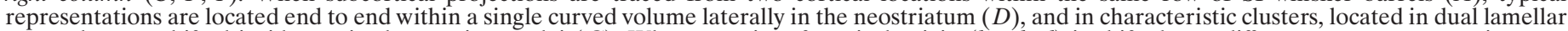

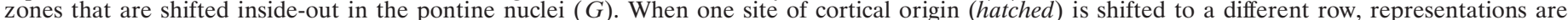

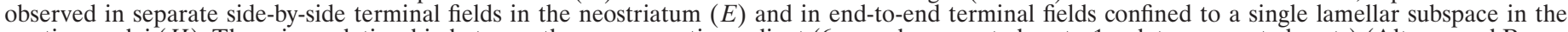

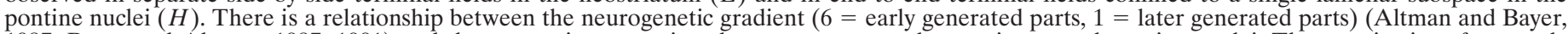

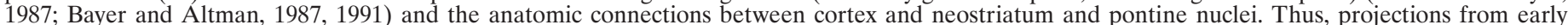

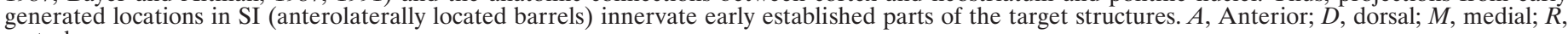
rostral.

the level of the pontine nuclei of pathways originating in individual adjacent cortical barrels ("open loop"). With increasing distance between cortical barrels, we found that the degree of overlap in the pontine nuclei gradually decreases (resembling more "closed loops"). In the basal ganglia circuitry, Alexander and coworkers (Alexander et al., 1986, 1990; Alexander and Crutcher, 1990) proposed that functional integration would take place within rather than among functionally related parallel circuits. In the neostriatum, such local integration is also indicated by the partially overlapping projections from individual SI whisker barrels (Alloway et al., 1999). It is less clear whether pontine projections from the SI whisker barrel cortex overlap with projections from cortical regions representing other modalities (Ruigrok and Cella, 1995; Brodal and Bjaalie, 1997; Schwarz and Thier, 1999), or to what degree terminal fields from subcortical sources of pontine afferents (Kosinski et al., 1986, 1988; Aas, 1989; Aas and Brodal, 1989; Lee and Mihailoff, 1990; Mihailoff, 1995) (for review, see Ruigrok and Cella, 1995; Allen and Hopkins, 1998; Liu and Mihailoff, 1999) overlap with projections from SI.

\section{Implications for cerebellar functions}

The organized SI-pontine topographic pattern reported in this study seems suitable for both specific integration (primarily of signals from neighboring whisker barrels, possibly also of other inputs, as discussed above) and segregation (of signals from widely separated whisker barrels). The pattern predicts that sequential activation of a column of whiskers (i.e., whiskers in the same location of different rows) leads to sequential activation of different neurons within the same lamellar subspace. Sequential activation of whiskers in the orthogonal direction (i.e., whiskers within the same row) would result in sequential activation of different lamellae in the pontine nuclei. Both spatial and temporal features of whisker activation would be preserved in the pontine nuclei and would thus presumably reach the cerebellum.

It is not obvious how this anatomic arrangement would directly relate to cerebellar function. Ivry (1997) has suggested that the cerebellum is specifically involved in the processing of timing information. It would appear that the SI-pontine projection pattern reported here is organized to preserve timing information in the movements of whiskers. A second theory of cerebellar function that would seem consistent with our findings is the sensory data acquisition hypothesis of Bower (1997a,b). This theory, which emerged from an analysis of the cerebellar perioral representations of tactile surfaces in the rat, proposes that the cerebellum is specifically involved in the control of sensory data acquisition. Furthermore, this theory proposes that the cerebro-pontocerebellar pathway is responsible for transferring information on the timing and context of cerebral cortically directed sensory acquisition movements and behaviors as well as information about 
the ongoing processing of sensory tactile data within the cerebral cortex. The organized pontine topography combined with local integration capabilities discussed above could represent a substrate for such information transfer.

\section{REFERENCES}

Aas J-E (1989) Subcortical projections to the pontine nuclei in the cat. J Comp Neurol 282:331-354.

Aas J-E, Brodal P (1989) Demonstration of a mamillo-ponto-cerebellar pathway a multi-tracer study. Eur J Neurosci 1:61-74.

Alexander GE, Crutcher MD (1990) Neural representations of the target (goal) of visually guided arm movements in three motor areas of the monkey. J Neurophysiol 64:164-178.

Alexander GE, DeLong MR, Strick PL (1986) Parallel organization of functionally segregated circuits linking basal ganglia and cortex. Annu Rev Neurosci 9:357-381.

Alexander GE, Crutcher MD, DeLong MR (1990) Basal gangliathalamocortical circuits: parallel substrates for motor, oculomotor, "prefrontal" and "limbic" functions. Prog Brain Res 85:119-146.

Allen GV, Hopkins DA (1998) Convergent prefrontal cortex and mamillary body projections to the medial pontine nuclei: a light and electron microscopic study in the rat. J Comp Neurol 398:347-358.

Alloway KD, Mutic JJ, Hoover JE (1998) Divergent corticostriatal projections from a single cortical column in the somatosensory cortex of rats. Brain Res 785:341-346.

Alloway KD, Crist J, Mutic JJ, Roy SA (1999) Corticostriatal projections from rat barrel cortex have an anisotropic organization that correlates with vibrissal whisking behavior. J Neurosci 19:10908-10922.

Altman J, Bayer SA (1978) Prenatal development of the cerebellar system in the rat. II. Cytogenesis and histogenesis of the inferior olive, pontine gray, and the precerebellar reticular nuclei. J Comp Neurol 179:49-75.

Altman J, Bayer SA (1987) Development of the precerebellar nuclei in the rat: IV. The anterior precerebellar extramural migratory stream and the nucleus reticularis tegmenti pontis and the basal pontine gray. J Comp Neurol 257:529-552.

Armstrong-James M, Millar J (1979) Carbon fibre microelectrodes. J Neurosci Methods 1:279-287.

Bajo VM, Merchan MA, Malmierca MS, Nodal FR, Bjaalie JG (1999) Topographic organization of the dorsal nucleus of the lateral lemniscus in the cat. J Comp Neurol 407:349-366.

Bayer SA, Altman J (1987) Directions in neurogenetic gradients and patterns of anatomical connections in the telencephalon. Prog Neurobiol 29:57-106

Bayer SA, Altman J (1991) Neocortical development. New York: Raven.

Berg BG, Almaas TJ, Bjaalie JG, Mustaparta H (1998) The macroglomerular complex of the antennal lobe in the tobacco budworm moth Heliothis virescens: specified subdivision in four compartments according to information about biologically significant compounds. J Comp Physiol [A] 183:669-682.

Bishop KM, Goudreau G, O'Leary DD (2000) Regulation of area identity in the mammalian neocortex by Emx2 and Pax6. Science 288:344-349.

Bjaalie JG, Brodal P (1989) Visual pathways to the cerebellum: segregation in the pontine nuclei of terminal fields from different visual cortica areas in the cat. Neuroscience 29:95-107.

Bjaalie JG, Leergaard TB (2000) Functions of the pontine nuclei in cerebro-cerebellar communication. Trends Neurosci 23:152.

Bjaalie JG, Daehlen M, Stensby TV (1997a) Surface modelling from biomedical data. In: Numerical methods and software tools in industrial mathematics (Daehlen M, Tveito A, eds), pp 9-26. Boston: Birkhauser.

Bjaalie JG, Sudbø J, Brodal P (1997b) Corticopontine terminal fibres form small scale clusters and large scale lamellae in the cat. NeuroReport 8:1651-1655.

Bower JM (1997a) Control of sensory data acquisition. Int Rev Neurobiol 41:489-513

Bower JM (1997b) Is the cerebellum sensory for motor's sake, or motor for sensory's sake: the view from the whiskers of a rat? Prog Brain Res 114:463-496.

Brodal P (1968) The corticopontine projection in the cat. I. Demonstration of a somatotopically organized projection from the primary sensorimotor cortex. Exp Brain Res 5:210-234.

Brodal P (1978) The corticopontine projection in the rhesus monkey. Origin and principles of organization. Brain 101:251-283.

Brodal P, Bjaalie JG (1992) Organization of the pontine nuclei. Neurosci Res 13:83-118.

Brodal P, Bjaalie JG (1997) Salient anatomic features of the cortico-pontocerebellar pathway. Prog Brain Res 114:227-249.

Brown LL (1992) Somatotopic organization in rat striatum: evidence for a combinational map. Proc Natl Acad Sci USA 89:7403-7407.

Brown LL, Smith DM, Goldblum LM (1998) Organizing principles of cortical integration in the rat neostriatum: somatotopic organization of sensorimotor activity. J Comp Neurol 392:468-499.

Erzurumlu RS, Jhaveri S (1992) Emergence of connectivity in the embryonic rat parietal cortex. Cereb Cortex 2:336-352.

Fabri M, Burton H (1991) Ipsilateral cortical connections of primary somatic sensory cortex in rats. J Comp Neurol 311:405-424.
Gerfen CR (1989) The neostriatal mosaic: striatal patch-matrix organization is related to cortical lamination. Science 246:385-388.

Graybiel AM (1990) Neurotransmitters and neuromodulators in the basal ganglia. Trends Neurosci 13:244-254.

He SQ, Dum RP, Strick PL (1993) Topographic organization of corticospinal projections from the frontal lobe: motor areas on the lateral surface of the hemisphere. J Neurosci 13:952-980.

Heimer L, Zahm DS, Alheid GF (1995) Basal ganglia. In: The rat nervous system (Paxinos G, ed), pp 579-628. San Diego: Academic.

Hicks SP, D'Amato CJ (1968) Cell migrations to the isocortex in the rat. Anat Rec 160:619-634.

Hoover JE, Strick PL (1999) The organization of cerebellar and basal ganglia outputs to primary motor cortex as revealed by retrograde transneuronal transport of herpes simplex virus type $1 . \mathrm{J}$ Neurosci 19:1446-1463.

Ivry R (1997) Cerebellar timing systems. Int Rev Neurobiol 41:555-573.

Kincaid AE, Wilson CJ (1996) Corticostriatal innervation of the patch and matrix in the rat neostriatum. J Comp Neurol 374:578-592.

Kincaid AE, Zheng T, Wilson CJ (1998) Connectivity and convergence of single corticostriatal axons. J Neurosci 18:4722-4731.

Kosinski RJ, Neafsey EJ, Castro AJ (1986) A comparative topographical analysis of dorsal column nuclear and cerebral cortical projections to the basilar pontine gray in rats. J Comp Neurol 244:163-173.

Kosinski RJ, Azizi SA, Mihailoff GA (1988) Convergence of cortico- and cuneopontine projections onto components of the pontocerebellar system in the rat: an anatomical and electrophysiological study. Exp Brain Res 71:541-556.

Lanciego JL, Wouterlood FG (1994) Dual anterograde axonal tracing with Phaseolus vulgaris-leucoagglutinin (PHA-L) and biotinylated dextran amine (BDA). Neuroscience Protocols 94-050-06-01-13.

Land PW, Simons DJ (1985) Cytochrome oxidase staining in the rat SmI barrel cortex. J Comp Neurol 238:225-235.

Lee HS, Mihailoff GA (1990) Convergence of cortical and cerebellar projections on single basilar pontine neurons: a light and electron microscopic study in the rat. Neuroscience 39:561-577.

Leergaard TB, Bjaalie JG (1995) Semi-automatic data acquisition for quantitative neuroanatomy. MicroTrace-computer programme for recording of the spatial distribution of neuronal populations. Neurosci Res 22:231-243.

Leergaard TB, Bjaalie JG (1998) From cortical 2-D to brain stem 3-D maps: organization of corticopontine projections in developing and adult rats. Soc Neurosci Abstr 24:262.9.

Leergaard TB, Lakke EA, Bjaalie JG (1995) Topographical organization in the early postnatal corticopontine projection: a carbocyanine dye and 3-D computer reconstruction study in the rat. J Comp Neurol 361:77-94.

Leergaard TB, Lyngstad KA, Thompson JH, Taeymans S, Vos BP, De Schutter E, Bower JM, Bjaalie JG (2000) Rat somatosensory cerebropontocerebellar pathways: spatial relationships of the somatotopic map of the primary somatosensory cortex are preserved in a three-dimensional clustered pontine map. J Comp Neurol 422:246-266.

Liu H, Mihailoff GA (1999) Hypothalamopontine projections in the rat: anterograde axonal transport studies utilizing light and electron microscopy. Anat Rec 255:428-451.

Malach R (1994) Cortical columns as devices for maximizing neuronal diversity. Trends Neurosci 17:101-104.

Malach R, Graybiel AM (1986) Mosaic architecture of the somatic sensory-recipient sector of the cat's striatum. J Neurosci 6:3436-3458.

Malmierca MS, Rees A, Le Beau FE, Bjaalie JG (1995) Laminar organization of frequency-defined local axons within and between the inferior colliculi of the guinea pig. J Comp Neurol 357:124-144.

Malmierca MS, Leergaard TB, Bajo VM, Bjaalie JG, Merchan MA (1998) Anatomic evidence of a three-dimensional mosaic pattern of tonotopic organization in the ventral complex of the lateral lemniscus in cat. J Neurosci 18:10603-10618.

McCandlish CA, Li CX, Waters RS (1993) Early development of the SI cortical barrel field representation in neonatal rats follows a lateral-tomedial gradient: an electrophysiological study. Exp Brain Res 92:369-374.

Middleton FA, Strick PL (2000) Basal ganglia and cerebellar loops: motor and cognitive circuits. Brain Res Rev 31:236-250.

Mihailoff GA (1995) Orthograde axonal transport studies of projections from the zona incerta and pretectum to the basilar pontine nuclei in the rat. J Comp Neurol 360:301-318.

Mihailoff GA, Burne RA, Woodward DJ (1978) Projections of sensorimotor cortex to the basilar pontine nuclei in the rat: an autoradiographic study. Brain Res 145:347-354.

Mihailoff GA, McArdle CB, Adams CE (1981) The cytoarchitecture, cytology, and synaptic organization of the basilar pontine nuclei in the rat. I. Nissl and Golgi studies. J Comp Neurol 195:181-201.

Mihailoff GA, Lee H, Watt CB, Yates R (1985) Projections to the basilar pontine nuclei from face sensory and motor regions of the cerebral cortex in the rat. J Comp Neurol 237:251-263.

Panto MR, Cicirata F, Angaut P, Parenti R, Serapide F (1995) The projection from the primary motor and somatic sensory cortex to the basilar pontine nuclei. A detailed electrophysiological and anatomical study in the rat. J Hirnforsch 36:7-19.

Parent A, Hazrati LN (1995) Functional anatomy of the basal ganglia. I. 
The cortico-basal ganglia-thalamo-cortical loop. Brain Res Rev 20:91-127.

Ruigrok TJ, Cella F (1995) Precerebellar nuclei and red nucleus. In: The rat nervous system (Paxinos G, ed), pp 277-308. San Diego: Academic. Schmahmann JD, Pandya DN (1997) The cerebrocerebellar system. Int Rev Neurobiol 41:31-60.

Schwarz C, Thier P (1995) Modular organization of the pontine nuclei: dendritic fields of identified pontine projection neurons in the rat respect the borders of cortical afferent fields. J Neurosci 15:3475-3489.

Schwarz C, Thier P (1999) Binding of signals relevant for action: towards a hypothesis of the functional role of the pontine nuclei. Trends Neurosc 22:443-451.

Schwarz C, Thier P (2000) Functions of the pontine nuclei in cerebrocerebellar communication. Trends Neurosci 23:152-153.

Smart IH (1984) Histogenesis of the mesocortical area of the mouse telencephalon. J Anat 138:537-552.

Vassbø K, Nicotra G, Wiberg M, Bjaalie JG (1999) Monkey somatosensory cerebrocerebellar pathways: uneven densities of corticopontine neurons in different body representations of areas $3 \mathrm{~b}, 1$, and 2 . J Comp Neurol 406:109-128.
Webster KE (1961) Cortico-striate interrelations in the albino rat. J Anat 95:532-544.

Welker C (1971) Microelectrode delineation of fine grain somatotopic organization of $(\mathrm{SmI})$ cerebral neocortex in albino rat. Brain Res 26:259-275.

Welker C (1976) Receptive fields of barrels in the somatosensory neocortex of the rat. J Comp Neurol 166:173-189.

Wiesendanger R, Wiesendanger M (1982) The corticopontine system in the rat. II. The projection pattern. J Comp Neurol 208:227-238.

Wong-Riley M (1979) Changes in the visual system of monocularly sutured or enucleated cats demonstrable with cytochrome oxidase histochemistry. Brain Res 171:11-28.

Woolsey TA, Van der Loos H (1970) The structural organization of layer IV in the somatosensory region (SI) of mouse cerebral cortex. The description of a cortical field composed of discrete cytoarchitectonic units. Brain Res 17:205-242.

Wright AK, Norrie L, Ingham CA, Hutton EA, Arbuthnott GW (1999) Double anterograde tracing of outputs from adjacent "barrel columns" of rat somatosensory cortex. Neostriatal projection patterns and terminal ultrastructure. Neuroscience 88:119-133. 